\title{
Blood Triglycerides Levels and Dietary Carbohydrate Indices in Healthy Koreans
}

\author{
Hye Sook Min', Ji Yeon Kang'², Joohon Sung', Mi Kyung Kim² \\ ${ }^{1}$ Department of Preventive Medicine, Graduate School of Public Health, Seoul National University, Seoul; ${ }^{2}$ Department of Preventive Medicine, \\ Hanyang University College of Medicine, Seoul, Korea
}

Objectives: Previous studies have obtained conflicting findings regarding possible associations between indices measuring carbohydrate intake and dyslipidemia, which is an established risk factor of coronary heart disease. In the present study, we examined crosssectional associations between carbohydrate indices, including the dietary glycemic index (Gl), glycemic load (GL), total amount of carbohydrates, and the percentage of energy from carbohydrates, and a range of blood lipid parameters.

Methods: This study included 1530 participants (554 men and 976 women) from 246 families within the Healthy Twin Study. We analyzed the associations using a generalized linear mixed model to control for familial relationships.

Results: Levels of the Apo B were inversely associated with dietary $\mathrm{Gl}, \mathrm{GL}$, and the amount of carbohydrate intake for men, but these relationships were not significant when fat-adjusted values of the carbohydrate indices were used. Triglyceride levels were positively associated with dietary $\mathrm{Gl}$ and GL in women, and this pattern was more notable in overweight participants (body mass index [BMI] $\geq$ $\left.25 \mathrm{~kg} / \mathrm{m}^{2}\right)$. However, total, low-density lipoprotein and high-density lipoprotein cholesterol levels were not significantly related with carbohydrate intake overall.

Conclusions: Of the blood lipid parameters we investigated, only triglyceride levels were positively related with dietary carbohydrate indices among women participants in the Healthy Twin Study, with an interactive role observed for BMI. However, these associations were not observed in men, suggesting that the association between blood lipid levels and carbohydrate intake depends on the type of lipid, specific carbohydrate indices, gender, and BMI.

Key words: Carbohydrate, Glycemic index, Glycemic load, Dyslipidemias, Triglycerides

Received: February 2, 2016 Accepted: April 22, 2016

Corresponding author: Mi Kyung Kim, PhD

222 Wangsimni-ro, Seongdong-gu, Seoul 04763, Korea

Tel: +82-2-2220-0667, Fax: +82-2-2293-0660

E-mail:kmkkim@hanyang.ac.kr

This is an Open Access article distributed under the terms of the Creative Commons Attribution Non-Commercial License (http://creativecommons.org/licenses/bync/3.0/) which permits unrestricted non-commercial use, distribution, and reproduction in any medium, provided the original work is properly cited.

\section{INTRODUCTION}

The glycemic index $(\mathrm{Gl})$ is a relative ranking of the postprandial blood glucose level for each carbohydrate-containing food when compared to $50 \mathrm{~g}$ of glucose or a reference food, such as white bread, and the glycemic load (GL) of a food is a measure of the quantity $(\mathrm{g})$ of a carbohydrate in addition to its quality (GI). Previous studies have shown that a high dietary $\mathrm{GL}$, rather than $\mathrm{Gl}$, was significantly associated with an increased risk of coronary heart disease (CHD) [1-4]. However, conflicting results have been reported regarding the relationships of dietary Gl and GL with blood lipid profiles, which are 
established risk factors for CHD. It has been reported that higher dietary GI and GL values were associated with higher blood triglyceride and lower high-density lipoprotein (HDL) cholesterol levels $[5,6]$, but some recent systematic reviews have argued that the effect of a low Gl or GL diet was limited to lowering total and low-density lipoprotein (LDL) cholesterol levels, with no effect on triglycerides and HDL cholesterol [79]. Moreover, only a small amount of data has been published on associations between $\mathrm{Gl}, \mathrm{GL}$, and apolipoprotein levels, which are also targets of lipid-lowering treatments [10]. Overall, the dietary recommendations for low-GI and low-GL diets still lack sufficient evidence [11].

For East Asian populations, evidence regarding the effects of dietary $\mathrm{Gl}$ and $\mathrm{GL}$ on blood lipids remains mixed and inconclusive, as in Western populations. A few studies have shown that a higher dietary GL was associated with unfavorable levels of LDL cholesterol, triglycerides, and HDL cholesterol $[12,13]$, while others have argued that both dietary GI and GL were adversely linked with triglycerides and HDL cholesterol $[14,15]$. The results from these studies should be interpreted carefully, as dietary $\mathrm{Gl}$ and $\mathrm{GL}$ tend to be negatively correlated with dietary fat intake [12-15]. Accordingly, these results suggest that the effect of carbohydrate indices on blood lipids is greatly influenced by fat intake, and controlling for fat intake in statistical models is therefore crucial. The substitution model between carbohydrate and fat intake expressed as the percentage of total energy intake is an alternative way of explaining associations between the intake of different nutrients, providing additional information about which dietary factors may improve blood lipid levels [16,17].

As dyslipidemia is one of the major risk factors of CHD, lifestyle changes affecting blood lipid levels, such as diet, are important for the prevention of CHD. The previous inconsistent results regarding associations of dietary $\mathrm{Gl}$ and $\mathrm{GL}$ with blood lipid profiles may have been caused by uncontrolled differences in lifestyles, genetic factors, the effect of fat intake, or the study designs. The Healthy Twin Cohort used in the present study has incorporated data from adult same-sex twins and their families since 2005, ultimately including 1530 Korean healthy adults. We examined cross-sectional correlations of the dietary $\mathrm{Gl}, \mathrm{GL}$, total amount of carbohydrate intake, and percentage of energy from carbohydrates with blood lipid levels in this cohort, under the assumption that the participants' dietary habits were relatively stable and that the measured Gl and GL values reflected their individual dietary patterns. We controlled for familial relationships in the statistical analysis by using a generalized linear mixed model (GLMM).

\section{METHODS}

\section{Subjects}

The participants were 3479 enrollees in the Healthy Twin Study, which is a cohort of healthy adult same-sex twins ( $\geq 30$ years of age) and their immediate family members; the protocol of this study has been described in detail elsewhere [18]. Individuals with a self-reported history of dyslipidemia or lipidlowering therapy ( $n=219)$, self-reported history of cardiovascular disease $(n=61)$, stroke $(n=52)$, diabetes $(n=183)$, or hypertension $(n=446)$ were excluded because their dietary behavior may have been affected by disease. Individuals with implausibly low ( $<500 \mathrm{kcal}$ ) or high ( $>5000 \mathrm{kcal}$ ) total daily energy intakes, with incomplete information in their sociodemographic questionnaires, or who did not provide a blood sample were additionally excluded. The present study ultimately included 1530 participants (554 men, 976 women) from 246 families. All study participants provided written informed consent, and the study procedures were approved by the institutional review boards of the participating institutions.

\section{Clinical Characteristics and Biochemical Tests}

The following characteristics were assessed using a self-administered questionnaire: smoking status (current, past or never-smoker), alcohol consumption (regular drinker or not), regular exercise (yes or no), menopausal status (yes or no), average monthly household income (low, $<1.5$ million Korean won [KRW]/mo; middle, 1.5-3.0 million KRW/mo; high, >3.0 million KRW/mo) and duration of formal education ( $<9$ years, 9-11 years, $>12$ years). Body weight was measured to the nearest $0.1 \mathrm{~kg}$ using a digital scale (Tanita Co., Seoul, Korea), and height to the nearest $0.1 \mathrm{~cm}$ using a stadiometer (Samwha Co., Seoul, Korea). The body mass index (BMI) was calculated as the weight $(\mathrm{kg})$ divided by the squared height $\left(\mathrm{m}^{2}\right)$ and was used as a continuous variable for statistical analysis. After a 12-hour overnight fast, blood samples were collected and analyzed in a central laboratory authorized by the Korea Association of Quality Control for Clinical Laboratory Examinations. The biochemical tests included total cholesterol (enzymatic assay), HDL and LDL cholesterol (homogenous and enzymatic assays), triglycerides (enzymatic assay), Apo A1 and Apo B (enzymatic assays) on a Siemens ADVIA 1650 (Erlangen, 
Germany) and a Hitachi 7600-210/7180 biochemistry analyzer (Tokyo, Japan) using commercially available kits.

\section{Dietary Assessment}

Dietary intake was assessed using a semi-quantitative 103item food frequency questionnaire, which was developed based on the Korea Health and Nutrition Examination Survey and has been validated [19]. All participants were asked about the average frequency and portion sizes of each food item during a year. Food intake and nutrient intake per day were calculated using the recipe and nutrient database provided by the Korean Nutrition Association (Can Pro 3.0 database).

The Gl for each food item was determined using the International Tables of Glycemic Index (reference substance, glu- cose; GI for glucose = 100) [20] and a list of the Gls of Korean foods [21]. The GL of each food was calculated by multiplying the carbohydrate content in each serving by the $\mathrm{Gl}$ of that food, thereby reflecting both the quantity and the quality of carbohydrate content in a food. In order to calculate the dietary $G L$, all the $G L$ values for each food consumed over the course of one day were added together. The overall dietary GI was obtained by dividing the dietary GL by the total amount of carbohydrate intake per day. Nutrient intake levels, including carbohydrate intake, dietary Gl, dietary GL, protein intake, fat intake, cholesterol intake, and fiber intake were transformed to total energy-adjusted values, according to the residual method [22]. In addition, carbohydrate intake, dietary $\mathrm{Gl}$, and dietary GL were transformed to both total energy and

Table 1. Baseline characteristics of all participants from the Healthy Twin Study

\begin{tabular}{|c|c|c|c|}
\hline Variables & Total $(n=1530)$ & Male $(n=554)$ & Female $(n=976)$ \\
\hline Age (y) & $41.5 \pm 11.4$ & $42.4 \pm 12.3$ & $40.9 \pm 10.8$ \\
\hline BMI $\left(\mathrm{kg} / \mathrm{m}^{2}\right)$ & $23.3 \pm 3.2$ & $24.4 \pm 3.0$ & $22.7 \pm 3.2$ \\
\hline Education (>12 yr) & $620(40.5)$ & $257(46.4)$ & $363(37.2)$ \\
\hline Income (high) & $153(10.0)$ & $53(9.6)$ & 100 (10.3) \\
\hline Exercise (regular) & $525(34.3)$ & $213(38.5)$ & $312(32.0)$ \\
\hline Smoking (current) & $277(18.1)$ & $230(41.5)$ & $47(4.8)$ \\
\hline Alcohol (regular) & $1011(66.1)$ & $440(79.4)$ & $571(58.5)$ \\
\hline Menopause (yes) & - & - & $310(31.7)$ \\
\hline \multicolumn{4}{|l|}{ Dietary factors ${ }^{1}$} \\
\hline Glycemic index & $55.5 \pm 4.5$ & $55.6 \pm 4.7$ & $55.4 \pm 4.5$ \\
\hline Glycemic load & $178.1 \pm 65.2$ & $187.6 \pm 66.1$ & $172.7 \pm 64.1$ \\
\hline Total energy (kcal/d) & $1864.5 \pm 664.4$ & $1974.9 \pm 647.5$ & $1801.8 \pm 665.9$ \\
\hline Carbohydrate (g/d) & $319.0 \pm 114.2$ & $334.2 \pm 113.5$ & $310.3 \pm 113.7$ \\
\hline Protein (g/d) & $64.4 \pm 28.7$ & $68.0 \pm 27.6$ & $62.4 \pm 29.1$ \\
\hline Fat $(\mathrm{g} / \mathrm{d})$ & $35.1 \pm 21.2$ & $38.3 \pm 21.2$ & $33.3 \pm 21.0$ \\
\hline Cholesterol (mg/d) & $196.9 \pm 141.4$ & $205.1 \pm 137.1$ & $192.2 \pm 143.7$ \\
\hline Fiber $(\mathrm{g} / \mathrm{d})$ & $6.0 \pm 3.4$ & $5.8 \pm 3.1$ & $6.0 \pm 3.5$ \\
\hline $\begin{array}{l}\text { Percentage of energy from } \\
\text { carbohydrate }\end{array}$ & $68.6 \pm 8.7$ & $67.6 \pm 9.1$ & $69.1 \pm 8.4$ \\
\hline Percentage of energy from protein & $13.8 \pm 3.1$ & $13.8 \pm 3.2$ & $13.7 \pm 3.1$ \\
\hline Percentage of energy from fat & $16.8 \pm 6.8$ & $17.4 \pm 7.1$ & $16.4 \pm 6.7$ \\
\hline \multicolumn{4}{|l|}{ Blood lipids (mg/dL) } \\
\hline HDL cholesterol & $51.0 \pm 12.9$ & $47.1 \pm 11.9$ & $53.1 \pm 12.9$ \\
\hline LDL cholesterol & $108.1 \pm 30.3$ & $112.5 \pm 31.1$ & $105.6 \pm 29.6$ \\
\hline Total cholesterol & $185.4 \pm 34.6$ & $188.8 \pm 35.4$ & $183.5 \pm 34.0$ \\
\hline Triglycerides & $105.3 \pm 67.1$ & $131.4 \pm 77.6$ & $90.6 \pm 55.1$ \\
\hline Apo A1 & $153.4 \pm 26.0$ & $148.4 \pm 25.9$ & $156.2 \pm 25.7$ \\
\hline Apo B & $83.5 \pm 26.2$ & $91.8 \pm 27.8$ & $78.9 \pm 24.1$ \\
\hline
\end{tabular}

Values are presented as number (\%) or mean \pm standard deviation.

$\mathrm{BMI}$, body mass index; HDL, high-density lipoprotein; LDL, low-density lipoprotein.

${ }^{1}$ Before transformation by the residual method. 
fat intake-adjusted values using the same method.

\section{Statistical Analysis}

Continuous variables (age, BMI, lipid profiles, and nutrient intake values) are expressed as the means \pm standard error, and categorical variables (education, income, menopausal status, regular exercise, smoking, and alcohol) as frequencies or percentages. The variables were compared using analysis of variance or linear regression for continuous variables and the chi-square test for categorical variables. The relationships between dietary carbohydrates (dietary Gl, dietary GL, the amount of carbohydrates [g], and the percentage of total energy obtained from carbohydrates) and blood lipids were ana- lyzed using a GLMM to control the influence of familial relationships on the distribution of each lipid parameter. Additionally, we used total energy- and fat intake-adjusted values of dietary $\mathrm{Gl}$, dietary $\mathrm{GL}$, and the amount of carbohydrates $(\mathrm{g}$ ) in the GLMM instead of directly adjusting for fat intake in GLMM, due to the strong correlations of dietary $\mathrm{GL}$ and the amount of carbohydrate intake with fat intake. We used covariates as fixed effects in multivariate analyses, including age, BMI, education, alcohol, smoking, menopausal status, total energy intake, cholesterol intake, and fiber intake. Model 1 was adjusted for age, model 2 for confounders obtained from the univariate analyses, and model 3 for the confounders of model 2 and additional potential confounders. We used a substitution model

Table 2. Characteristics of variables according to the dietary Gl and GL (by quintile) in men ( $n=554)$

\begin{tabular}{|c|c|c|c|c|c|c|c|c|}
\hline & & Dietary GI & & & & Dietary GL & & - \\
\hline & 01 & 03 & 05 & p-value & 01 & 03 & 05 & p-value \\
\hline Age (y) & $43.4 \pm 11.6$ & $41.2 \pm 12.2$ & $45.4 \pm 14.2$ & 0.03 & $39.6 \pm 11.7$ & $42.4 \pm 11.3$ & $46.7 \pm 13.5$ & $<0.001$ \\
\hline BMI $\left(\mathrm{kg} / \mathrm{m}^{2}\right)$ & $24.2 \pm 2.8$ & $24.6 \pm 3.2$ & $24.2 \pm 2.7$ & 0.74 & $24.4 \pm 2.9$ & $24.5 \pm 3.1$ & $24.1 \pm 2.7$ & 0.63 \\
\hline Education (y) & & & & 0.007 & & & & 0.06 \\
\hline$<9$ & $8(11.0)$ & $10(13.7)$ & 29 (39.7) & & $7(9.6)$ & $13(17.8)$ & 29 (39.7) & \\
\hline $9-11$ & $45(20.1)$ & $46(20.5)$ & $35(15.6)$ & & $55(24.6)$ & $38(17.0)$ & $42(18.7)$ & \\
\hline$>12$ & $42(16.3)$ & 59 (22.9) & $46(17.9)$ & & $53(20.6)$ & $55(21.4)$ & $43(16.7)$ & \\
\hline Income & & & & 0.47 & & & & 0.17 \\
\hline Low & 83 (19.9) & 81 (19.4) & $86(20.6)$ & & $84(20.1)$ & 81 (19.4) & $91(21.8)$ & \\
\hline Middle & $6(7.2)$ & $22(26.5)$ & $15(18.1)$ & & 16 (19.3) & $17(20.5)$ & $15(18.1)$ & \\
\hline High & $6(11.3)$ & $12(22.6)$ & $9(17.0)$ & & $15(28.3)$ & $8(15.1)$ & $8(15.1)$ & \\
\hline Exercise & & & & 0.82 & & & & 0.61 \\
\hline No & $58(17.0)$ & $61(17.9)$ & $71(20.8)$ & & 76 (22.3) & $63(18.50)$ & 69 (20.2) & \\
\hline Yes & $37(17.4)$ & $54(25.4)$ & $39(18.3)$ & & 39 (18.3) & $43(20.2)$ & $45(21.1)$ & \\
\hline Smoking & & & & 0.14 & & & & 0.12 \\
\hline Never & $21(13.4)$ & 30 (19.1) & 36 (22.9) & & 31 (19.8) & 26 (16.6) & $33(21.0)$ & \\
\hline Former & $28(16.7)$ & 37 (22.2) & $29(17.4)$ & & $30(17.9)$ & $29(17.4)$ & 43 (25.8) & \\
\hline Current & $46(20.0)$ & $48(20.8)$ & $45(19.6)$ & & $54(23.5)$ & $51(22.2)$ & $38(16.5)$ & \\
\hline Alcoholic consumption & & & & 0.36 & & & & 0.01 \\
\hline Non-regular & $19(16.5)$ & $23(20.0)$ & $27(23.5)$ & & $21(18.2)$ & $21(18.2)$ & $41(35.6)$ & \\
\hline Regular & $76(17.3)$ & $93(21.1)$ & 83 (18.8) & & $94(21.4)$ & $85(19.3)$ & 74 (16.8) & \\
\hline Nutrient intake ${ }^{1}$ & & & & & & & & \\
\hline Total energy (kcal/d) & $1893.9 \pm 848.0$ & $2138.2 \pm 623.2$ & $1778.5 \pm 510.9$ & $<0.001$ & $1911.4 \pm 825.7$ & $1932.1 \pm 680.3$ & $2191.5 \pm 511.7$ & 0.003 \\
\hline Carbohydrate (g/d) & $276.6 \pm 62.2$ & $313.6 \pm 28.9$ & $346.2 \pm 23.0$ & $<0.001$ & $254.9 \pm 44.1$ & $321.1 \pm 15.4$ & $352.7 \pm 16.9$ & $<0.001$ \\
\hline Protein $(\mathrm{g} / \mathrm{d})$ & $80.0 \pm 22.1$ & $63.8 \pm 9.4$ & $53.4 \pm 7.1$ & $<0.001$ & $83.9 \pm 17.2$ & $61.9 \pm 7.0$ & $52.4 \pm 6.4$ & $<0.001$ \\
\hline Fat $(g / d)$ & $50.0 \pm 20.4$ & $36.5 \pm 10.1$ & $24.5 \pm 8.4$ & $<0.001$ & $57.1 \pm 14.4$ & $34.1 \pm 5.4$ & $22.1 \pm 6.1$ & $<0.001$ \\
\hline Cholesterol (mg/d) & $307.8 \pm 181.4$ & $188.3 \pm 87.1$ & $125.2 \pm 59.4$ & $<0.001$ & $328.6 \pm 156.5$ & $178.3 \pm 67.4$ & $120.0 \pm 65.4$ & $<0.001$ \\
\hline Fiber $(\mathrm{g} / \mathrm{d})$ & $8.1 \pm 3.5$ & $5.3 \pm 1.7$ & $4.0 \pm 1.1$ & $<0.001$ & $6.7 \pm 3.5$ & $5.3 \pm 2.2$ & $4.7 \pm 1.7$ & $<0.001$ \\
\hline
\end{tabular}

Values are presented as number (\%) or mean \pm standard deviation.

$p$-value was obtained using the linear regression analysis or analysis of variance, after adjusting for age (except for the age variable).

$\mathrm{Gl}$, glycemic index; GL, glycemic load; 0 , quintile; BMI, body mass index.

${ }^{1}$ After transformation by the residual method (except total energy). 
by including total energy intake, the percentage of energy obtained from carbohydrates and proteins, and other covariates in the GLMM $[16,17]$. By excluding the percentage of energy obtained from fat, the model presented the difference in $\mathrm{Gl}$ and $G L$ associated with the percentage of energy obtained from carbohydrates at the expense of the percentage of energy obtained from fat, while keeping the total energy intake and the percentage of energy obtained from protein constant. Finally, we stratified our analyses of the effects of dietary carbohydrates according to BMI ( $\geq 25 \mathrm{~kg} / \mathrm{m}^{2}$ vs. $<25 \mathrm{~kg} / \mathrm{m}^{2}$ ) to assess the interactive role of BMI. All analyses were conducted using SAS version 9.2 (SAS Institute Inc., Cary, NC, USA) and $p$ values $<0.05$ were considered to indicate statistical significance.

\section{RESULTS}

Summarized descriptive data classified by gender are presented in Table 1. The men showed a higher dietary GL and amount of carbohydrate intake, but the percentage of energy

Table 3. Characteristics of variables according to the dietary $\mathrm{Gl}$ and $\mathrm{GL}$ (by quintile) in women $(\mathrm{n}=976)$

\begin{tabular}{|c|c|c|c|c|c|c|c|c|}
\hline & \multicolumn{3}{|c|}{ Dietary GI } & \multirow{2}{*}{$p$-value } & \multicolumn{3}{|c|}{ Dietary GL } & \multirow{2}{*}{$p$-value } \\
\hline & 01 & 03 & 05 & & 01 & 03 & 05 & \\
\hline Age (y) & $41.1 \pm 10.4$ & $39.5 \pm 10.3$ & $43.1 \pm 12.1$ & 0.01 & $39.6 \pm 9.7$ & $41.3 \pm 11.4$ & $44.2 \pm 11.4$ & $<0.001$ \\
\hline $\mathrm{BMI}\left(\mathrm{kg} / \mathrm{m}^{2}\right)$ & $22.5 \pm 2.9$ & $22.6 \pm 2.8$ & $23.1 \pm 3.8$ & 0.19 & $22.6 \pm 3.2$ & $22.7 \pm 3.0$ & $23.6 \pm 3.7$ & 0.002 \\
\hline Education (y) & & & & 0.03 & & & & 0.50 \\
\hline$<9$ & $36(21.7)$ & 27 (16.3) & $41(24.7)$ & & 32 (19.3) & $30(18.1)$ & 43 (25.9) & \\
\hline $9-11$ & 87 (19.5) & $97(21.7)$ & $96(21.5)$ & & 80 (17.9) & 85 (19.0) & $97(21.7)$ & \\
\hline$>12$ & $87(24.0)$ & $66(18.2)$ & $58(16.0)$ & & $77(21.2)$ & $84(23.1)$ & $52(14.3)$ & \\
\hline Income & & & & 0.06 & & & & 0.32 \\
\hline Low & $161(22.4)$ & $140(19.4)$ & $148(20.6)$ & & $148(20.6)$ & $141(19.6)$ & $144(20.0)$ & \\
\hline Middle & $23(14.7)$ & $28(18.0)$ & 31 (19.8) & & 20 (12.8) & 41 (26.3) & $32(20.5)$ & \\
\hline High & $26(26.0)$ & $22(22.0)$ & $16(16.0)$ & & $21(21.0)$ & $17(17.0)$ & $16(16.0)$ & \\
\hline Menopause & & & & 0.66 & & & & 0.002 \\
\hline No & $142(19.5)$ & $141(21.4)$ & $123(18.0)$ & & $137(20.6)$ & $132(19.8)$ & $114(17.1)$ & \\
\hline Yes & $68(21.0)$ & $49(15.8$ & $72(23.2)$ & & $52(16.7)$ & $67(21.6)$ & $78(25.2)$ & \\
\hline Exercise & & & & 0.95 & & & & 0.25 \\
\hline No & $137(20.6)$ & $128(19.3)$ & $130(19.6)$ & & $128(19.3)$ & $139(20.9)$ & $136(20.52)$ & \\
\hline Yes & $73(23.4)$ & 62 (19.9) & 65 (20.8) & & $61(19.6)$ & $60(19.2)$ & $56(17.9)$ & \\
\hline Smoking & & & & 0.99 & & & & 0.10 \\
\hline Never & $193(21.6)$ & 168 (18.8) & $180(20.2)$ & & $167(18.7)$ & $186(20.8)$ & $180(20.2)$ & \\
\hline Former & $6(16.7)$ & $5(13.9)$ & $7(19.4)$ & & $9(25.0)$ & $7(19.4)$ & $3(8.3)$ & \\
\hline Current & $11(23.4)$ & $17(36.2)$ & $8(17.0)$ & & $13(27.6)$ & $6(12.8)$ & $9(19.2)$ & \\
\hline Alcoholic consumption & & & & 0.57 & & & & 0.01 \\
\hline Non-regular & $88(21.7)$ & $75(18.5)$ & $85(20.9)$ & & 74 (18.2) & $72(17.8)$ & $102(25.2)$ & \\
\hline Regular & $122(19.8)$ & $115(20.8)$ & $110(19.4)$ & & $115(20.1)$ & $127(22.2)$ & $90(15.8)$ & \\
\hline \multicolumn{9}{|l|}{ Nutrient intake ${ }^{1}$} \\
\hline Total energy (kcal/d) & $1904.6 \pm 889.4$ & $1887.3 \pm 590.5$ & $1607.7 \pm 457.4$ & $<0.001$ & $1854.3 \pm 910.4$ & $1704.3 \pm 635.8$ & $2024.5 \pm 537.3$ & $<0.001$ \\
\hline Carbohydrate $(\mathrm{g} / \mathrm{d})$ & $296.2 \pm 50.4$ & $322.3 \pm 30.5$ & $347.8 \pm 25.2$ & $<0.001$ & $265.7 \pm 38.7$ & $329.9 \pm 17.5$ & $358.4 \pm 17.4$ & $<0.001$ \\
\hline Protein $(\mathrm{g} / \mathrm{d})$ & $76.4 \pm 18.6$ & $62.7 \pm 8.8$ & $54.0 \pm 8.2$ & $<0.001$ & $83.7 \pm 15.1$ & $60.5 \pm 6.9$ & $52.4 \pm 5.8$ & $<0.001$ \\
\hline Fat $(\mathrm{g} / \mathrm{d})$ & $44.2 \pm 16.8$ & $33.9 \pm 10.6$ & $24.1 \pm 9.2$ & $<0.001$ & $53.5 \pm 13.6$ & $32.0 \pm 5.7$ & $20.4 \pm 6.0$ & $<0.001$ \\
\hline Cholesterol (mg/d) & $266.0 \pm 153.3$ & $190.1 \pm 76.9$ & $131.6 \pm 84.8$ & $<0.001$ & $325.5 \pm 154.2$ & $182.5 \pm 72.8$ & $108.1 \pm 61.3$ & $<0.001$ \\
\hline Fiber $(\mathrm{g} / \mathrm{d})$ & $8.8 \pm 3.9$ & $5.8 \pm 1.7$ & $4.2 \pm 1.2$ & $<0.001$ & $7.8 \pm 4.2$ & $5.8 \pm 2.1$ & $5.3 \pm 1.9$ & $<0.001$ \\
\hline
\end{tabular}

Values are presented as number (\%) or mean \pm standard deviation.

$p$-value was obtained using the linear regression analysis or analysis of variance, after adjusting for age (except for the age variable).

$\mathrm{Gl}$, glycemic index; GL, glycemic load; $\mathrm{Q}$, quintile; BMI, body mass index.

${ }^{1}$ After transformation by the residual method (except total energy). 


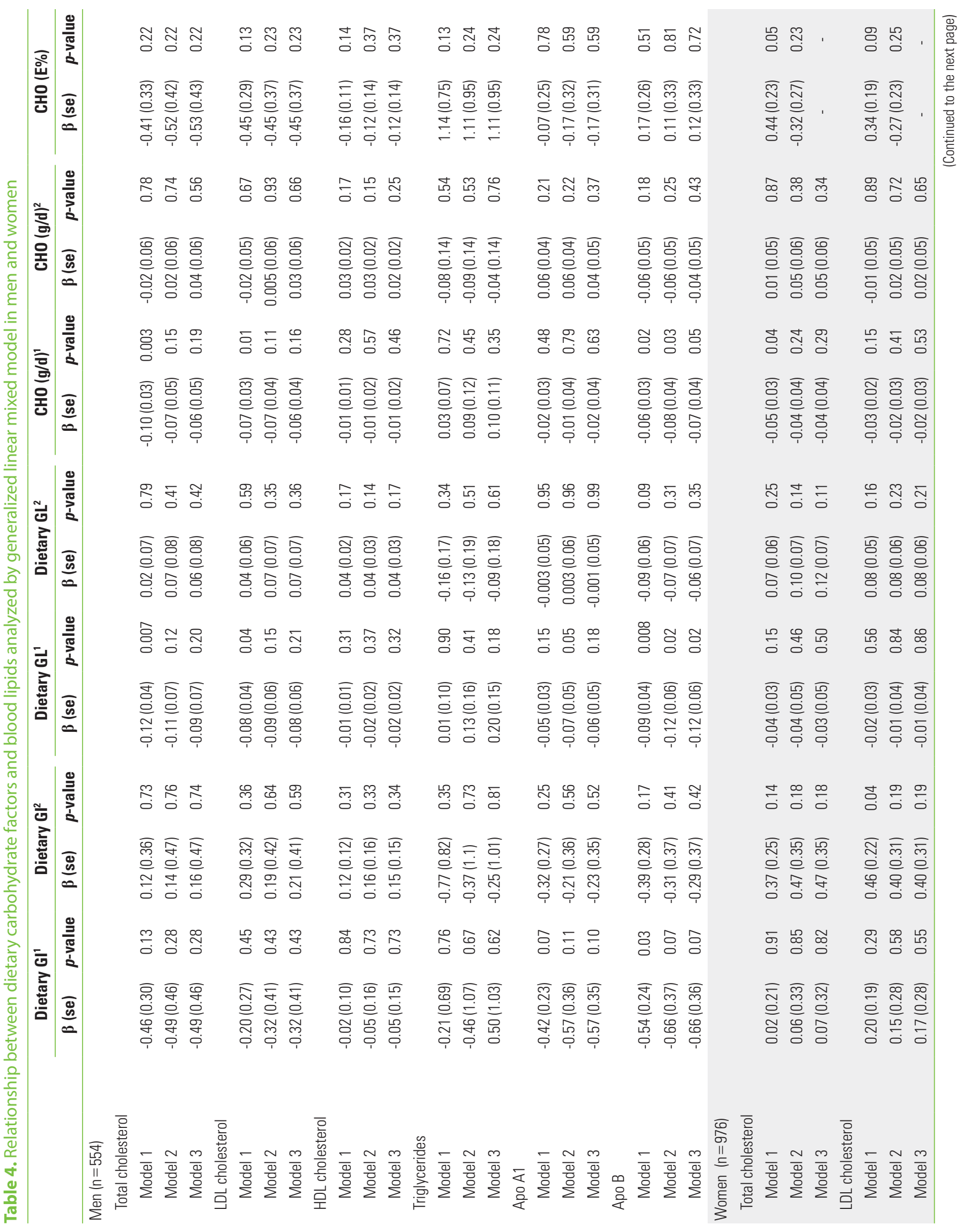




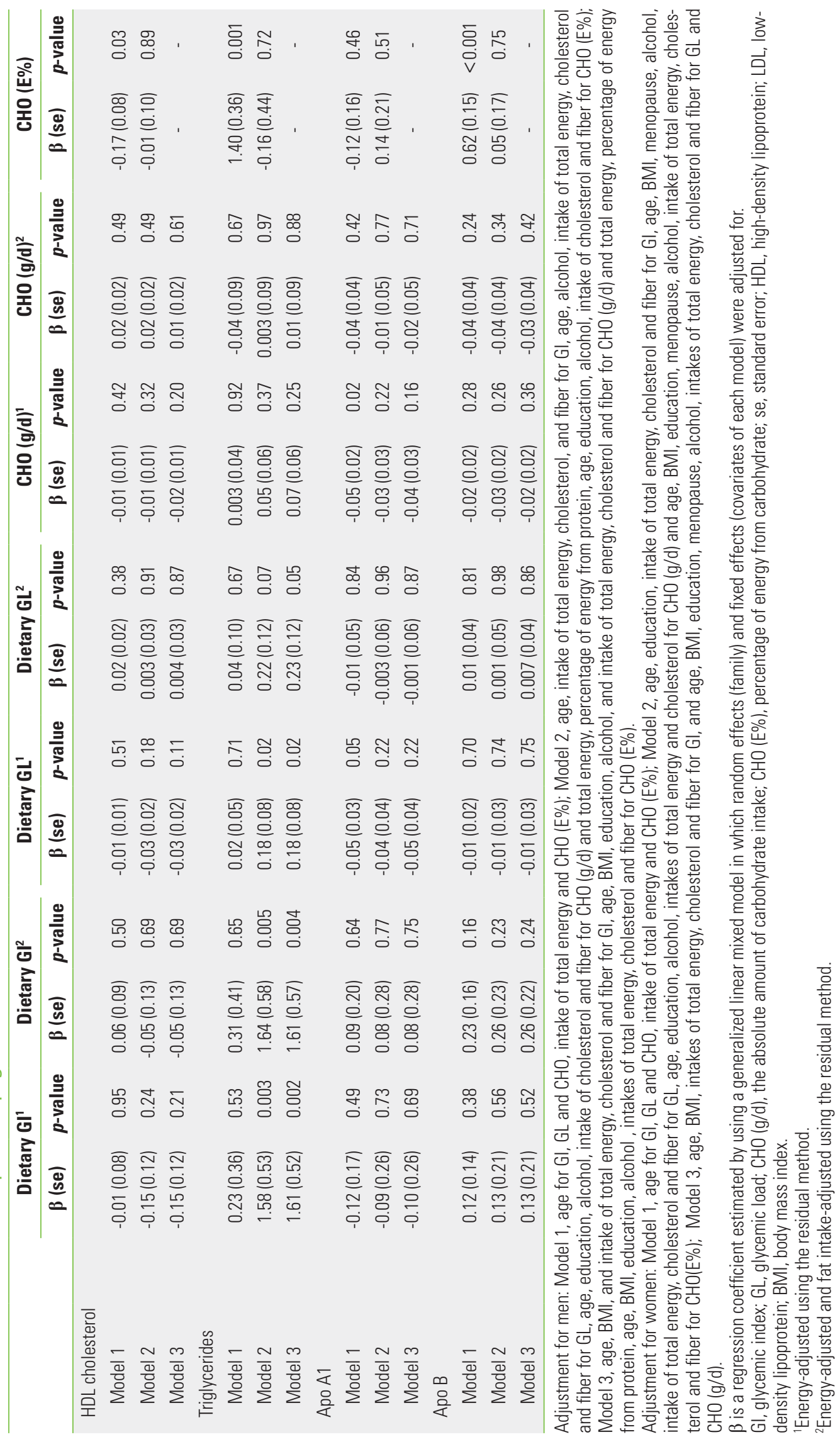




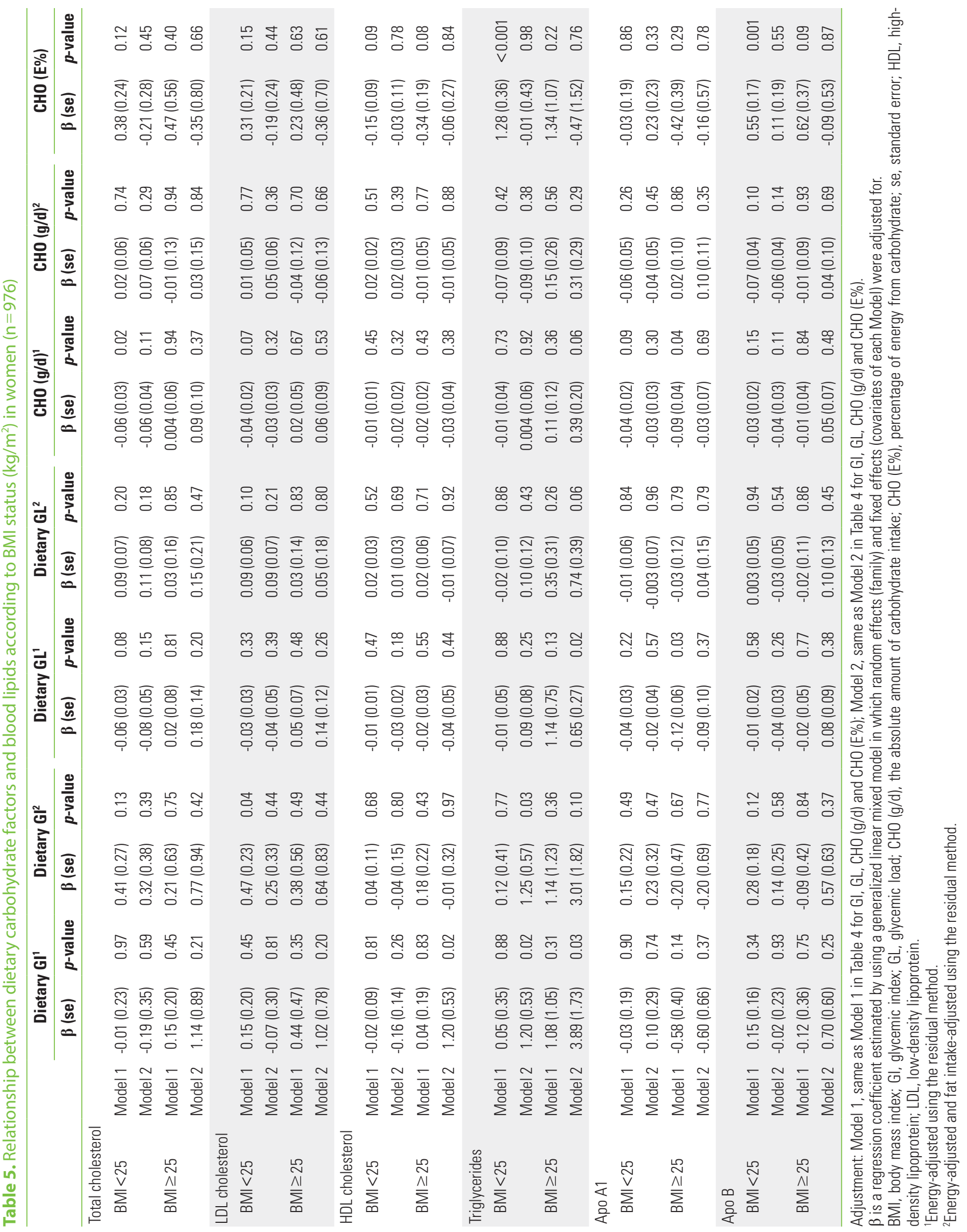


from carbohydrates was not higher than was observed for women. The dietary $\mathrm{Gl}$ was not significantly different between men and women ( $55.6 \pm 4.7$ vs. $55.4 \pm 4.5$ ). All blood lipid profiles of men, except for HDL cholesterol and Apo A1, were higher than those of women.

\section{Variables Associated With Dietary Glycemic In- dex and Glycemic Load}

The relationships of demographic variables with dietary $\mathrm{Gl}$ and GL in quintiles are shown in Tables 2 and 3. Older participants showed a higher dietary $\mathrm{Gl}$ and $\mathrm{GL}$, both for men $(p=0.03)$ and for women $(p<0.001)$. BMI was not associated with either dietary $\mathrm{Gl}$ or $\mathrm{GL}$ for either gender, with the exception of dietary $\mathrm{GL}$ for women $(p<0.001)$. Years of education showed a consistent inverse relationship with dietary $\mathrm{Gl}$ and $\mathrm{GL}(p=0.08$ and $p=0.006$ for men, $p=0.04$ and $p=0.01$ for women, respectively). Among men, current smokers and regular alcohol drinkers tended to show a lower dietary $G L$ than former or never-smokers $(p=0.04)$ and non-regular drinkers $(p=0.01)$. Menopausal women had a higher GL than premenopausal women $(p<0.01)$, and women who regularly drank alcohol had a lower $\mathrm{GL}$ than non-regular drinkers $(p<0.001)$. Total energy intake was inversely associated with dietary GI ( $p<0.001$ for men, $p<0.01$ for women) but positively associated with dietary $\mathrm{GL}$ ( $p<0.01$ for men, $p<0.001$ for women). The intake of protein, fat, fiber, and cholesterol decreased as the dietary Gl and GL increased ( $p<0.001$ for all nutrients) for both genders. Fat intake was negatively correlated with the amount of carbohydrate intake $(r=-0.97$ for men, $r=-0.96$ for women), as well as dietary $G L$ ( $r=-0.92$ for men, $r=-0.90$ for women) and $\mathrm{Gl}$ ( $r=-0.62$ for men, $r=-0.51$ for women). After adjusting the carbohydrate indices for fat, the negative correlations with fat intake were attenuated to $r=-0.24$ (men) and $r=-0.28$ (women) for absolute carbohydrate intake, $r=-0.21$ (men) and $r=-0.23$ (women) for dietary $G L$, and $r=-0.11$ (men) and $\mathrm{r}=-0.09$ (women) for $\mathrm{Gl}$.

\section{Relationship Between Indices of Dietary Carbo- hydrates and Blood Lipids}

In GLMM assessing the relationship between carbohydrate indices and blood lipids for men (Table 4), Apo B was negatively associated with dietary $\mathrm{Gl}$ ( $p=0.025$, model 1$)$, dietary $\mathrm{GL}$ ( $p=0.008$ in model $1, p=0.02$ in model 2 ) and the absolute amount of carbohydrate intake ( $p=0.02$ in model $1, p=0.03$ in model 2). However, these associations disappeared when the fat intake-adjusted values for dietary $\mathrm{Gl}, \mathrm{GL}$ and the amount of carbohydrate intake $(\mathrm{g} / \mathrm{d})$ were used in the models. Moreover, total cholesterol and LDL cholesterol were related to the dietary $\mathrm{GL}$ and the amount of carbohydrate intake $(\mathrm{g} / \mathrm{d})$, but these associations were only found in model 1 . The replacement of fat intake with carbohydrates, as expressed as percentage of energy, was not significantly associated with blood lipid parameters in men. Most lipid levels tended to decrease as carbohydrates were substituted for fats in a participant's total dietary intake, but triglyceride levels increased; however, these associations did not reach statistical significance.

In women, triglyceride levels were positively associated with dietary $\mathrm{Gl}$ ( $p=0.003$ in model $2, p=0.002$ in model 3 ) and fat intake-adjusted values of dietary $\mathrm{Gl}$ ( $p=0.005$ in model 2, $p=0.004$ in model 3) (Table 4). The dietary GL was also positively associated with triglyceride levels ( $p=0.04$ in model 2 , $p=0.02$ in model 3), and fat intake-adjusted values of the dietary $\mathrm{GL}$ showed a positive but weaker relationship with triglyceride levels ( $p=0.06$ in model $2, p=0.05$ in model 3 ). Total cholesterol, LDL cholesterol, and Apo A1 were associated with the amount of carbohydrate intake, dietary $\mathrm{Gl}$ and $\mathrm{GL}$, but these findings were limited to model 1. The replacement of fat intake with carbohydrates was positively associated with triglyceride $(p<0.001)$ and Apo B $(p<0.001)$ levels, and negatively related with $\mathrm{HDL}$ cholesterol $(p=0.03)$ but only in model 1 .

As some reports have found an interactive role of obesity on the relationship between dietary carbohydrates and blood lipids $[5,15]$, the participants were stratified by BMI (BMI $\geq 25 \mathrm{~kg} /$ $\mathrm{m}^{2}$ and $\mathrm{BMI}<25 \mathrm{~kg} / \mathrm{m}^{2}$ ) for subgroup analysis (Table 5). For women, triglycerides were significantly associated with dietary $\mathrm{Gl}$ in both overweight participants ( $n=201,20.6 \%$; $\beta=3.89 ; p=0.03)$ and non-overweight participants ( $\mathrm{n}=775$, $79.4 \% ; \beta=1.20, p=0.02$ ) in multivariate analyses, but the $\beta$-value of overweight participants was more than three times greater than that of non-overweight participants. Although this was not a statistically significant finding, the $\beta$-values for overweight participants were greater than those for nonoverweight participants for dietary GL, the amount of carbohydrate intake, and the fat intake-adjusted values of both indices. In contrast, among men, triglyceride levels were not related with dietary $\mathrm{Gl}$ and $\mathrm{GL}$ in overweight participants (38.4\%, $\mathrm{n}=213 ; \beta=-0.03 ; p=0.98$ for $\mathrm{Gl}$ and $\beta=0.16 ; p=0.57$ for $\mathrm{GL}$ ) and non-obese participants (61.6\%, $\mathrm{n}=341 ; \beta=1.62 ; p=0.19$ for $\mathrm{Gl}$ and $\beta=0.35 ; p=0.06$ for $\mathrm{GL}$ ). In contrast to the results from women, the $\beta$-values of overweight participants were 
even smaller than those of non-overweight participants for $\mathrm{Gl}$ and $\mathrm{GL}$, although the results were not statistically significant (data not shown).

\section{DISCUSSION}

The present study showed that triglyceride levels were positively associated with dietary carbohydrate intake in women, and that BMI may have played an interactive role in this association. In men, Apo B was inversely associated with dietary $\mathrm{Gl}$, $\mathrm{GL}$, and the amount of carbohydrate intake, but these relationships were not evident when carbohydrate indices were adjusted for fat intake. Meanwhile, total cholesterol, LDL cholesterol, and HDL cholesterol levels were not significantly related with carbohydrate intake overall.

In light of the fact that fat intake markedly decreased as dietary $\mathrm{Gl}$ and $\mathrm{GL}$ increased in the current study, it was notable that blood triglyceride levels were positively associated with dietary Gl and GL in women even before fat-adjustment (Table 5). Although it was not statistically significant, greater carbohydrate intake was also linked with higher triglyceride levels in the substitution model. Regarding the link between fat intake and blood lipid levels, the Pearson's correlation coefficients were positive overall for both men and women $(r=0.009-0.14$, data not shown in Results) but for triglycerides it was negative ( $r=-0.05$ for men, $r=-0.01$ for women). This further supports the conclusion that greater carbohydrate intake was associated with higher triglyceride levels, as fat intake was negatively correlated with dietary $\mathrm{Gl}$ and $\mathrm{GL}$ in our study. This association is in accordance with previous results $[9,11,23]$. Sacks et al. [24] recently reported that low-GI and low-carbohydrate diets did not favorably affect HDL cholesterol and LDL cholesterol levels, but did lower triglyceride levels considerably. A gender difference has been found in some studies of Asian populations, in which either the Gl or GL was significantly linked with triglyceride levels for women but not for men $[13,15,25]$. It is likely that hormonal differences underlie these results [26]. However, in the present study, a significant relationship between triglycerides and GI/GL was found in postmenopausal women $(n=310,31.7 \%)$ but this relationship was rather weak in premenopausal women (data not shown).

In our study, triglyceride levels were especially elevated as $\mathrm{Gl}$ or GL increased in overweight participants in contrast to non-overweight participants. The interactive role of BMI status on this association in the current study is in accordance with previous results $[5,15]$. Insulin resistance has been associated with high triglyceride levels $[27,28]$ and it has been recognized that a high level of insulin resistance is present in the obese population [29], meaning that insulin resistance may play an important role in mediating the higher triglyceride levels observed in overweight individuals with a high $\mathrm{Gl}$ or $\mathrm{GL}$.

Apolipoproteins are important constituents of lipoproteins and a therapeutic target for the prevention of CHD. One molecule of Apo B binds to each lipoprotein, including LDL, and therefore it reflects lipoprotein density and is highly correlated with non-HDL cholesterol levels [30]. Only a few results have been published on the relationship between Apo B and carbohydrate intake, with one report showing that a Mediterranean-style low-GL diet lowered Apo B levels in comparison to the control group [10]. Our results demonstrating an inverse relationship between Apo B levels and carbohydrate intake may have been affected by the negative correlation between fat intake and carbohydrate intake, because the association was not apparent when we used fat-adjusted values for $\mathrm{Gl}, \mathrm{GL}$, and the amount of carbohydrate intake.

The present study has some weaknesses, particularly due to the presence of strong co-linearity between fat intake and indices of carbohydrate intake. For this reason, we attempted to adjust for fat intake in the process of transforming $\mathrm{Gl}, \mathrm{GL}$, and the amount of carbohydrate intake using the residual method. Doing so reversed the direction of the associations between carbohydrate indices and blood lipid parameters (Tables 4 and 5); however, a negative correlation between fat intake and indices of carbohydrate was still present. Other residual confounders may have been present in the mixed model that we used to control for familial relationships, and it was possible that the variables reflecting environmental and genetic factors were partially controlled for. Incorporating a larger population and using quantitative metrics to measure regular exercise, smoking and alcohol consumption would facilitate a more detailed analysis.

In summary, our results did not show clear associations between carbohydrate indices and levels of total cholesterol, LDL cholesterol, and HDL cholesterol. However, triglyceride levels were significantly associated with the dietary $\mathrm{Gl}$ and $\mathrm{GL}$ for women, and these findings were replicated in models using fat-adjusted values. Dietary carbohydrate intake may differentially affect blood lipids depending on the type of lipid, gender, and BMI. 


\section{CONFLICT OF INTEREST}

The authors have no conflicts of interest associated with the material presented in this paper.

\section{ORCID}

Hye Sook Min http://orcid.org/0000-0003-0029-5163

Joohon Sung http://orcid.org/0000-0001-9948-0160

Mi Kyung Kim http://orcid.org/0000-0001-8503-2631

\section{REFERENCES}

1. Liu S, Willett WC, Stampfer MJ, Hu FB, Franz M, Sampson L, et al. A prospective study of dietary glycemic load, carbohydrate intake, and risk of coronary heart disease in US women. Am J Clin Nutr 2000;71(6):1455-1461.

2. Fan J, Song Y, Wang Y, Hui R, Zhang W. Dietary glycemic index, glycemic load, and risk of coronary heart disease, stroke, and stroke mortality: a systematic review with meta-analysis. PLoS One 2012;7(12):e52182.

3. Yu D, Shu XO, Li H, Xiang YB, Yang G, Gao YT, et al. Dietary carbohydrates, refined grains, glycemic load, and risk of coronary heart disease in Chinese adults. Am J Epidemiol 2013;178(10): 1542-1549.

4. Sieri S, Krogh V, Berrino F, Evangelista A, Agnoli C, Brighenti F, et al. Dietary glycemic load and index and risk of coronary heart disease in a large italian cohort: the EPICOR study. Arch Intern Med 2010;170(7):640-647.

5. Liu S, Manson JE, Stampfer MJ, Holmes MD, Hu FB, Hankinson $\mathrm{SE}$, et al. Dietary glycemic load assessed by food-frequency questionnaire in relation to plasma high-density-lipoprotein cholesterol and fasting plasma triacylglycerols in postmenopausal women. Am J Clin Nutr 2001;73(3):560-566.

6. Ma Y, Li Y, Chiriboga DE, Olendzki BC, Hebert JR, Li W, et al. Association between carbohydrate intake and serum lipids. J Am Coll Nutr 2006;25(2):155-163.

7. Ludwig DS. The glycemic index: physiological mechanisms relating to obesity, diabetes, and cardiovascular disease. JAMA 2002;287(18):2414-2423.

8. Goff LM, Cowland DE, Hooper L, Frost GS. Low glycaemic index diets and blood lipids: a systematic review and metaanalysis of randomised controlled trials. Nutr Metab Cardiovasc Dis 2013;23(1):1-10.

9. Fleming P, Godwin M. Low-glycaemic index diets in the man- agement of blood lipids: a systematic review and meta-analysis. Fam Pract 2013;30(5):485-491.

10. Jones JL, Comperatore M, Barona J, Calle MC, Andersen C, MCIntosh $\mathrm{M}$, et al. A Mediterranean-style, low-glycemic-load diet decreases atherogenic lipoproteins and reduces lipoprotein (a) and oxidized low-density lipoprotein in women with metabolic syndrome. Metabolism 2012;61(3):366-372.

11. Kristo AS, Matthan NR, Lichtenstein AH. Effect of diets differing in glycemic index and glycemic load on cardiovascular risk factors: review of randomized controlled-feeding trials. Nutrients 2013;5(4):1071-1080.

12. Choi H, Song S, Kim J, Chung J, Yoon J, Paik HY, et al. High carbohydrate intake was inversely associated with high-density lipoprotein cholesterol among Korean adults. Nutr Res 2012;32(2):100-106.

13. Nakashima M, Sakurai M, Nakamura K, Miura K, Yoshita K, Morikawa $Y$, et al. Dietary glycemic index, glycemic load and blood lipid levels in middle-aged Japanese men and women. J Atheroscler Thromb 2010;17(10):1082-1095.

14. Murakami K, Sasaki S, Takahashi Y, Okubo H, Hosoi Y, Horiguchi $\mathrm{H}$, et al. Dietary glycemic index and load in relation to metabolic risk factors in Japanese female farmers with traditional dietary habits. Am J Clin Nutr 2006;83(5):1161-1169.

15. Kim K, Yun SH, Choi BY, Kim MK. Cross-sectional relationship between dietary carbohydrate, glycaemic index, glycaemic load and risk of the metabolic syndrome in a Korean population. Br J Nutr 2008;100(3):576-584.

16. Faerch K, Lau C, Tetens I, Pedersen OB, Jørgensen T, BorchJohnsen $\mathrm{K}$, et al. A statistical approach based on substitution of macronutrients provides additional information to models analyzing single dietary factors in relation to type 2 diabetes in danish adults: the Inter99 study. J Nutr 2005;135(5):11771182.

17. Similä ME, Kontto JP, Männistö S, Valsta LM, Virtamo J. Glycaemic index, carbohydrate substitution for fat and risk of $\mathrm{CHD}$ in men. Br J Nutr 2013;110(9):1704-1711.

18. Sung J, Cho SI, Lee K, Ha M, Choi EY, Choi JS, et al. Healthy Twin: a twin-family study of Korea--protocols and current status. Twin Res Hum Genet 2006;9(6):844-848.

19. Ahn Y, Kwon E, Shim JE, Park MK, Joo Y, Kimm K, et al. Validation and reproducibility of food frequency questionnaire for Korean genome epidemiologic study. Eur J Clin Nutr 2007;61 (12):1435-1441.

20. Foster-Powell K, Holt SH, Brand-Miller JC. International table of glycemic index and glycemic load values: 2002. Am J Clin 
Nutr 2002;76(1):5-56.

21. Lee JS, Lee JS, Yang CB, Shin HK. Blood glucose response to some cereals and determination of their glycemic index to rice as standard food. Korean J Nutr 1997;30(10):1170-1179 (Korean).

22. Willett WC, Howe GR, Kushi LH. Adjustment for total energy intake in epidemiologic studies. Am J Clin Nutr 1997;65(4 Suppl):1220S-1228S.

23. Westman EC, Feinman RD, Mavropoulos JC, Vernon MC, Volek JS, Wortman JA, et al. Low-carbohydrate nutrition and metabolism. Am J Clin Nutr 2007;86(2):276-284.

24. Sacks FM, Carey VJ, Anderson CA, Miller ER 3rd, Copeland T, Charleston J, et al. Effects of high vs low glycemic index of dietary carbohydrate on cardiovascular disease risk factors and insulin sensitivity: the OmniCarb randomized clinical trial. JAMA 2014;312(23):2531-2541.

25. Song S, Lee JE, Song WO, Paik HY, Song Y. Carbohydrate intake and refined-grain consumption are associated with metabolic syndrome in the Korean adult population. J Acad Nutr Diet 2014; 114(1):54-62.

26. Power ML, Schulkin J. Sex differences in fat storage, fat metabolism, and the health risks from obesity: possible evolutionary origins. Br J Nutr 2008;99(5):931-940.

27. Bonora E, Capaldo B, Perin PC, Del Prato S, De Mattia G, Frittitta $L$, et al. Hyperinsulinemia and insulin resistance are independently associated with plasma lipids, uric acid and blood pressure in non-diabetic subjects. The GISIR database. Nutr Metab Cardiovasc Dis 2008;18(9):624-631.

28. Reaven G. Insulin resistance and coronary heart disease in nondiabetic individuals. Arterioscler Thromb Vasc Biol 2012;32(8):1754-1759.

29. Ye J. Mechanisms of insulin resistance in obesity. Front Med 2013;7(1):14-24.

30. Walldius G, Jungner I. Apolipoprotein B and apolipoprotein $A-I$ : risk indicators of coronary heart disease and targets for lipid-modifying therapy. J Intern Med 2004;255(2):188-205. 